\section{Funcionalidade em pessoas idosas atendidas no centro de atendimento comunitário do UniCEUB (CAC-UniCEUB)*}

\section{Facts about human functioning of elderly assessed at UniCEUB community health care center}

Jaquelyne Bernard da Silva ${ }^{1}$ Linconl Agudo Oliveira Benito ${ }^{2}$
* Recebido em: 29/08/2016. Aprovado em: 04/10/2017.

1 Graduanda em Enfermagem do Centro Universitário de Brasília (UniCEUB).

2 Docente do Centro Universitário de Brasília (UniCEUB).

\section{Resumo}

Trata-se de um estudo de coorte, transversal, analítico e quantitativo, que utilizou o Índex de Katz, proposto por Sidney Katz em 1998. Num total de 49 idosos avaliados, o perfil dos atores sociais participantes deste estudo se constituiu de 53,05\% $(\mathrm{n}=26)$ pessoas do sexo feminino, $97,95 \%(\mathrm{n}=48)$ brasileiros, $87,75 \%(\mathrm{n}=43)$ negam tabagismo, 81,65\% ( $\mathrm{n}=40)$ negam etilismo, 42,85\% ( $\mathrm{n}=21)$ possuem ensino fundamental, 49\% $(\mathrm{n}=24)$ realizam exercícios físicos, 55,10\% ( $\mathrm{n}=27)$ possuem sono normal, $69,40 \%(\mathrm{n}=34)$ possuem entre 2 e 4 filhos, $14,30 \%$ $(\mathrm{n}=07)$ moram, respectivamente, juntamente às cidades satélites do Gama (RA-II) e São Sebastião (RA-XIV), $24,50 \%(\mathrm{n}=12)$ possuem renda de 02 a 04 salários, $28,55 \%(n=14)$ realizam caminhada, $51 \%(n=25)$ eram da região nordeste. Foi verificado que $95,90 \%(n=47)$ adquiriram 06 enquanto pontuação e $4,10 \%(\mathrm{n}=02)$ adquiriram 05 , se caracterizando respectivamente enquanto idosos independentes por conseguirem realizar as atividades de banhar-se, vestir-se, ir ao banheiro, transferência, continência e alimentação.

Palavras-chave: Envelhecimento. Idoso. Avaliação geriátrica. Atividades cotidianas.

\begin{abstract}
A cohort cross-sectional analytic and quantitative study was carried on, applying Katz index, proposed by Sidney Katz in 1988. A total of 49 elderly were assessed, among which $53.05 \%(n=26)$; were females; 97.95\% ( $\mathrm{n}=48)$ were Brazilian, $87.75 \%(\mathrm{n}=43)$ claimed not to smoke; $81.65 \%(\mathrm{n}=40)$, claimed not to drink and alcoholic drinks, $42.85 \%(\mathrm{n}=21)$ finished Junior High School; 49\% ( $\mathrm{n}=24)$ exercise; 55.10\% $(\mathrm{n}=27)$ claimed to sleep normally; $69.40 \%(\mathrm{n}=34)$ have 2 to 4 children; $14.30 \%(\mathrm{n}=07)$ live respectively in the towns of Gama (Administrative Region, AR- II), and São Sebastião (AR-XIV), 24.5\% ( $\mathrm{n}=12)$ had an income ranging from 2 to 4 base wage salaries; $28.55 \%(n=14)$ go on regular walks, and $51 \%(n=25)$ were originally from Brazil's northeastern states. As a $95.90 \%(n=47 \%)$ scored 06 on the assessment procedures, while the remaining $4.1 \%(\mathrm{n}=2)$ scored 05. consequence, they were classified as independent elderly, as they were able to perform activities such as: taking a shower, dressing up, using a toilet, locomotion, urinary continence, and eating.
\end{abstract}

Keywords: Aging. Elderly. Geriatric assessment. Ordinary activities. 


\section{Introdução}

Segundo a OMS, até 2025, o Brasil será o sexto $\left(6^{\circ}\right)$ país do mundo em número de idosos. Assim, ainda, é ampla a desinformação sobre essa população e as peculiaridades e desafios que o envelhecimento populacional desencadeará em nosso contexto social. Entre os anos 1980 e 2000, a população com 60 anos ou mais cresceu 7,3 milhões, totalizando mais de 14,5 milhões em 2000 (BRASIL, 2010; OPAS, 2005).

Atenta às mudanças e às transformações relacionadas ao aumento populacional de idosos na sociedade, a nação Brasileira propôs a Política Nacional do Idoso (PNI), o Estatuto do Idoso, dentre outras legislações e dispositivos, assegurando seus direitos sociais, autonomia, integração e a sua participação efetiva na sociedade. Assim, o envelhecimento populacional se constitui enquanto fenômeno natural, mundial e irreversível, caracterizado pela mudança na faixa etária da população (BRASIL, 2010; POLITICA NACIONAL DO IDOSO, 2010; ESTATUTO DO IDOSO, 2007).

Nesse sentido, o processo de envelhecimento é definido enquanto um conjunto de modificações morfológicas, fisiológicas, bioquímicas e psicológicas, que caracterizam a perda progressiva da capacidade de adaptação do ser vivo ao meio ambiente, sendo considerado um processo dinâmico e progressivo. A redução das funções orgânicas, apresentadas durante o envelhecimento, tendem a aumentar com o tempo, com um ritmo que varia não somente de um órgão para outro, mas entre pessoas idosas da mesma idade. Essas alterações no processo de envelhecimento devem-se às condições de vida desiguais e de trabalho, a que estiveram submetidas as pessoas idosas (PINHO et al, 2012; SANTOS; GRIEP, 2011; FERREIRA et al, 2010).

As transformações decorrentes do processo de envelhecimento são evidenciadas pela diminuição da força muscular, amplitude de movimento, da velocidade de contração muscular, da acuidade visual e auditiva, e pelas alterações posturais que influenciam a mobilidade funcional e o deficit de equilíbrio em idosos, podendo ser relacionada aos sistemas músculo-esquelético, neuromuscular e sensorial. Essas transformações podem ocasionar prejuízos, como o aumento no risco de quedas, a redução do nível de independência funcional e a diminuição na qualidade de vida (QV) (PINHO et al, 2012; SANTOS; GRIEP, 2011; MOREIRA et al, 2005).
Devido ao aumento do envelhecimento populacional, a transição demográfica, epidemiológica e nutricional é caracterizada pelo aumento de doenças crônicas degenerativas e a diminuição das doenças infecto-contagiosas, resultando no aumento da demanda dessa população por serviços de saúde, ocasionando transformações que nem sempre são acompanhadas de mudanças no atendimento às necessidades de saúde desse grupo populacional (GUERRA; CALDAS, 2010; MOREIRA et al, 2005).

A avaliação geriátrica e gerontológica, relacionas às informações coletadas na história clínica, no exame físico, com aquelas obtidas por um conjunto de instrumentos específicos, permite a detecção de incapacidades, além da possibilidade de planejar cuidados prolongados, avaliar a gravidade da doença e avaliar o progresso do paciente. Os instrumentos de avaliação geriátrica que mais se destacam são aqueles que avaliam a pessoa idosa do ponto de vista cognitivo (GUERRA; CALDAS, 2010; LOURENÇO; VERAS, 2006).

Alguns estudos revelam que cerca de $40 \%$ das pessoas, com 65 anos ou mais de idade, precisam de algum tipo de ajuda para realizar pelo menos uma tarefa como fazer compras, cuidar das finanças, preparar refeições e limpar a casa. Nesse sentido, cerca de $10 \%$ requer auxílio para realizar tarefas básicas, como tomar banho, vestir-se, ir ao banheiro, alimentar-se, sentar e levantar de cadeiras e camas. Esses dados remetem à preocupação por mais de seis (6) milhões de pessoas e famílias, e a um e meio (milhão de idosos gravemente fragilizados no Brasil (GUERRA; CALDAS, 2010; LOURENÇO; VERAS, 2006; KARSCH, 2003).

Em consequência do aumento do número de idosos, há necessidade de instrumentos de avaliação funcional. O Índex de Independência nas Atividades de Vida Diária (AVDs), desenvolvido por Sidney Katz, é um dos instrumentos de avaliação mais antigos e mais citados na literatura nacional e internacional. Diversas publicações têm apresentado, no entanto, versões modificadas desse instrumento, dificultando aos leitores sua correta utilização (ALVES et al, 2007; DUARTE; ANDRADE; LEBRÃO, 2007).

Em torno de 1950, Katz e colaboradores desenvolveram seis (06) medidas das AVDs, que até hoje são utilizadas em vários estudos, procuram avaliar o grau de dependência de pessoas idosas. O Índice de Katz (IK) é o que possui validade e confiança comprovada entre as

1 
escalas que avaliam as AVDs. Com isso, ele permite avaliar a capacidade de autocuidado, identificar os fatores de risco e a das doenças diagnosticadas (PELEGRIN et al, 2008; DUARTE; ANDRADE; LEBRÃO, 2007).

A avaliação da capacidade funcional (CF) tem sido um instrumento útil para avaliar o estado de saúde dos idosos, devido a muitos deles terem várias doenças simultaneamente, que variam em severidade, provocando, normalmente, diferentes impactos na vida cotidiana. A incapacidade funcional foi definida pela Organização Mundial da Saúde (OMS); enquanto a dificuldade foi definida em virtude de uma deficiência, para realizar atividades típicas e pessoalmente desejadas na sociedade. Constantemente, é avaliada por meio de declaração indicativa de dificuldade, ou de necessidade de ajuda, em atividades básicas de cuidados pessoais e em atividades mais complexas, necessárias para ter autonomia na comunidade (PEREIRA et al, 2012; PARAHYBA; SIMÕES, 2006; OPAS, 2005).

Para avaliar a CF do idoso em realizar atividades que o permitam cuidar de si e viver independente, pode ser utilizada a Avaliação Funcional (AF) que se constitui enquanto um conjunto de dados clínicos, testes e escalas. São considerados enquanto parâmetros para a avaliação, o equilíbrio e mobilidade, a função cognitiva, as condições emocionais, a disponibilidade e adequação do suporte familiar e social, as condições ambientais e a capacidade para realizar as AVDs e as atividades instrumentais de vida diária (AIVD) (RODRIGUES et al., 2013; PEREIRA et al, 2012; ARAÚJO et al., 2010; NAKATANI et al, 2009; CAMARA et al., 2008; PEDRAZZI et al, 2007; PARAHYBA; SIMÕES, 2006; COSTA et al, 2006).

Nesse sentido, se constitui enquanto objetivo da presente pesquisa, analisar a questão da funcionalidade de pessoas idosas, atendidas no Centro de Atendimento Comunitário do Centro Universitário de Brasília (CAC-UniCEUB), no ano de 2015.

\section{Metodologia}

Trata-se de um estudo de Coorte, transversal e analítico, potencializado por uma abordagem quantitativa e que se propôs a analisar a questão da funcionalidade em pessoas idosas atendidas pelo "Centro de Atendimento Comunitário do Centro Universitário de Brasília (CAC-UniCEUB)" no recorte histórico formado pelo ano de "2015". Para fins organizacionais, a presente pesquisa considera enquanto pessoa idosa aquela que se encontra na faixa etária igual ou superior a sessenta (60) anos.

Para a aquisição de dados necessários, a edificação do presente estudo foi solicitada, previamente, autorização juntamente à Coordenação da Faculdade de Enfermagem do Centro Universitário de Brasília (UniCEUB). Objetivando respeitar, integralmente, os dispositivos expostos juntamente à resolução de número 466/2012 do Conselho Nacional de Saúde (CNS), relacionadas às diretrizes e às normas regulamentadoras em pesquisas utilizando seres humanos, um projeto de pesquisa foi submetido à apreciação e à analise bioética do Comitê de Ética e Pesquisa (CEP) do Centro Universitário de Brasília (UniCEUB), sendo este aprovado e obtendo o número da CAAE “50998015.9.0000.0023”.

Após a aquisição das autorizações para início do processo de pesquisa, foi identificado um universo de 56 prontuários. Destes, 12,05\% ( $\mathrm{n}=07$ ) não foram selecionados por não cumprirem os critérios de inclusão da presente pesquisa, possuírem registros pouco legíveis ou ilegíveis, ou que de alguma forma, inviabiliza-se a captação dos dados necessários a construção da presente pesquisa, conforme exposto junto a tabela de número 01 . Após esse processo, foram selecionados um universo de 49 prontuários, sendo estas classificadas enquanto fontes primárias.

Tabela 01 - Quantitativo de prontuários de pessoas idosas atendidas junto ao CAC-UniCEUB, Brasília, Distrito Federal, Brasil, $(n=56)$ :

\begin{tabular}{ccc}
\hline Universo de prontuários & Frequência & $\%$ \\
\hline Prontuários identificados & 56 & 100,00 \\
Prontuários selecionados & 49 & 87,50 \\
Prontuários não selecionados & 07 & 12,50 \\
\hline
\end{tabular}

Fonte: Produção dos autores do estudo, 2016.

Constituem-se enquanto critérios de inclusão da presente pesquisa prontuários de pessoas idosas que tenham recebido atendimento em saúde juntamente ao CAC do UniCEUB no ano de 2015, que se encontrem na faixa etária igual ou superior a sessenta (60) anos, que fossem tanto do sexo feminino quanto do sexo masculino, e que estejam em condições de participarem da presente pesquisa.

Associando informações teóricas e empíricas, Katz e equipe desenvolveram o denominado "Index of ADL (Index of Activity Daily Living)", um instrumento de medida das atividades de vida diária (AVD) hierarquicamente relacionado e organizado para mensurar 
independência no desempenho dessas seis funções. Esse instrumento representa a descrição de um fenômeno observado em um contexto biológico e social e, apesar do desenvolvimento de outros, ainda tem sido um dos mais utilizados na literatura gerontológica para avaliar a funcionalidade dos idosos em relação ao que hoje são denominadas Atividades Básicas de Vida Diária (KATZ; STROUD, 1989).

Segundo essa escala, os idosos eram classificados como independentes se eles desenvolvessem a atividade (qualquer das seis propostas) sem supervisão, orientação ou qualquer tipo de auxílio direto (KATZ et al., 1963). O Index de Independência nas Atividades de Vida Diária (AVDs) - Index of ADL, desenvolvido por Sidney Katz, é um instrumento de avaliação funcional muito utilizado na literatura gerontológica tanto em nível nacional quanto internacional (DUARTE; ANDRADE; LEBRÃO, 2007).

As fontes secundárias derivaram de levantamentos bibliográficos eletrônicos desenvolvidos juntamente à base de dados informatizados nacionais e internacionais, sendo estas a Biblioteca Virtual em Saúde (BVS), a Medical LiteratureAnalysisand Retrieval System Online (MEDLINE) e a ScientificElectronic Library Online (SciELO). Por meio desse processo, foram adquiridos artigos de periódicos científicos, manuais oficiais e legislação correlata.

Foram utilizados os Descritores em Ciências da Saúde (DeCS) da Biblioteca Virtual em Saúde (BVS) sendo estes, "Idoso" com o número de registro "20174" e identificador único "D000368", "Avaliação geriátrica" com o número de registro "24693" e descritor único “D015577”, "Envelhecimento" com o número de registro “22101” e descritor único “D000375” e "atividades cotidianas" com o número de registro "212" e identificador único “D000203”. No processo de conjugação dos descritores eleitos, foram utilizados os operadores lógicos booleanos "and", "or" e "andnot".

Os subsídios adquiridos foram organizados juntamente ao um banco de dados, utilizando o software Microsoft Excel $2013^{\circledR}$, pertencente ao pacote Microsoft Office $2013^{\circledR}$, for Windows ${ }^{\oplus}$. Os resultados foram expostos por meio da utilização de tabelas explicativas.

\section{Resultados e Discussão}

No decurso do processo de organização e análise dos dados, foi possível verificar que o perfil das pessoas idosas atendidas juntamente à instituição participante do estudo se constituiu de 53,05\% $(n=26)$ pessoas do sexo feminino, $61,20 \%(n=30)$ sexagenários com média de idade igual a 67,85 anos, $97,95 \%(n=48)$ brasileiros, $87,75 \%$ $(\mathrm{n}=43)$ negam tabagismo, 81,65\% $(\mathrm{n}=40)$ negam etilismo, $42,85 \%(n=21)$ possuem ensino fundamental, $49 \%$ $(n=24)$ negam restrição alimentar, 49\% $(n=24)$ realizam exercícios físicos, 65,30\% ( $\mathrm{n}=32)$ negam alergias, 53,05 $(\mathrm{n}=26)$ negam uso de adoçantes, $55,10 \%(\mathrm{n}=27)$ possuem sono normal, 69,40\% ( $\mathrm{n}=34)$ possuem entre 2 e 4 filhos, $14,30 \%$ ( $n=07)$ estão com média de filhos igual a 4,04, moram, respectivamente, nas cidades satélites do Gama (RA-II), São Sebastião (RA-XIV) e Sobradinho (RA-V), $24,50 \%(\mathrm{n}=12)$ possuem renda de 02 a 04 salários, 22,40\% $(n=11)$ eram naturais de Minas Gerais, 28,55\% $(n=14)$ realizam caminhada, $51 \%(n=25)$ eram da região nordeste, conforme exposto juntamente à tabela de número 2 .

Tabela 2 - Perfil socioeconômico de pessoas idosas atendidas no CAC-UniCEUB no ano de 2015, Brasília, Distrito Federal, Brasil, $(n=49)$ :

\begin{tabular}{|c|c|c|}
\hline Categorias & Frequência & $\%$ \\
\hline \multicolumn{3}{|l|}{ Gênero } \\
\hline Feminino & 26 & 53,05 \\
\hline Masculino & 23 & 46,95 \\
\hline \multicolumn{3}{|l|}{ Idade } \\
\hline 60 a 69 anos & 30 & 61,20 \\
\hline 70 a 79 & 19 & 38,80 \\
\hline \multicolumn{3}{|l|}{ Nacionalidade } \\
\hline Brasileira & 48 & 97,95 \\
\hline Estrangeira & 01 & 2,05 \\
\hline \multicolumn{3}{|l|}{ Tabagismo } \\
\hline Não & 43 & 87,75 \\
\hline Sim & 05 & 10,20 \\
\hline Não informado & 1 & 2,05 \\
\hline \multicolumn{3}{|l|}{ Etilismo } \\
\hline Não & 40 & 81,65 \\
\hline Sim & 08 & 16,30 \\
\hline Não informado & 01 & 2,05 \\
\hline \multicolumn{3}{|l|}{ Escolaridade } \\
\hline Ensino fundamental & 21 & 42,85 \\
\hline Ensino médio & 13 & 26,55 \\
\hline Ensino superior & 06 & 12,25 \\
\hline Não informado & 09 & 18,35 \\
\hline \multicolumn{3}{|l|}{ Restrição alimentar } \\
\hline Não & 24 & 49,00 \\
\hline Sim & 17 & 34,70 \\
\hline Não informado & 08 & 16,30 \\
\hline \multicolumn{3}{|l|}{ Exercícios físicos } \\
\hline Sim & 24 & 49,00 \\
\hline Não & 24 & 49,00 \\
\hline Não informado & 1 & 2,00 \\
\hline \multicolumn{3}{|l|}{ Alergias } \\
\hline Não & 32 & 65,30 \\
\hline Sim & 11 & 22,45 \\
\hline Não informado & 6 & 12,25 \\
\hline \multicolumn{3}{|l|}{ Adoçante } \\
\hline Não & 26 & 53,05 \\
\hline
\end{tabular}




\begin{tabular}{|c|c|c|c|}
\hline & Sim & 21 & 42,85 \\
\hline & Não informado & 2 & 4,10 \\
\hline \multicolumn{4}{|l|}{ Sono } \\
\hline & Normal & 27 & 55,10 \\
\hline & Dificuldade & 18 & 36,75 \\
\hline & Sonolência & 4 & 8,15 \\
\hline \multicolumn{4}{|c|}{ Quantitativo de filhos } \\
\hline & 01 & 02 & 4,10 \\
\hline & 02 a 04 & 34 & 69,40 \\
\hline & 05 a 09 & 07 & 14,30 \\
\hline & 10 ou mais & 04 & 8,10 \\
\hline & Nenhum ou não informado & 02 & 4,10 \\
\hline \multicolumn{4}{|c|}{ Região administrativa } \\
\hline & Gama & 07 & 14,30 \\
\hline & São Sebastião & 07 & 14,30 \\
\hline & Sobradinho & 07 & 14,30 \\
\hline & Guará & 05 & 10,15 \\
\hline & Paranoá & 02 & 4,05 \\
\hline & Samambaia & 02 & 4,05 \\
\hline & Águas claras & 01 & 2,05 \\
\hline & Candangolândia & 01 & 2,05 \\
\hline & Ceilândia & 01 & 2,05 \\
\hline & Cidade ocidental & 01 & 2,05 \\
\hline & Estrutural & 01 & 2,05 \\
\hline & Itapõa & 01 & 2,05 \\
\hline & Núcleo Bandeirante & 01 & 2,05 \\
\hline & Parkway & 01 & 2,05 \\
\hline & Planaltina & 01 & 2,05 \\
\hline & Sudoeste & 01 & 2,05 \\
\hline & Taguatinga & 01 & 2,05 \\
\hline & Valparaiso & 01 & 2,05 \\
\hline & Vicente Pires & 01 & 2,05 \\
\hline & Não informado ou outros & 06 & 12,20 \\
\hline \multicolumn{4}{|c|}{ Renda } \\
\hline & 01 salário & 07 & 14,30 \\
\hline & De 02 a 04 salários & 12 & 24,50 \\
\hline & De 05 a 07 salários & 04 & 8,15 \\
\hline & Não informado & 26 & 53,05 \\
\hline \multicolumn{4}{|c|}{ Naturalidade } \\
\hline & Minas Gerais & 11 & 22,45 \\
\hline & Bahia & 07 & 14,30 \\
\hline & Goiás & 05 & 10,20 \\
\hline & Piauí & 05 & 10,20 \\
\hline & Ceará & 04 & 8,15 \\
\hline & Paraíba & 03 & 6,10 \\
\hline & Pernambuco & 03 & 6,10 \\
\hline & Maranhão & 02 & 4,05 \\
\hline & Alagoas & 01 & 2,05 \\
\hline & Mato Grosso & 01 & 2,05 \\
\hline & Mato Grosso do Sul & 01 & 2,05 \\
\hline & Pará & 01 & 2,05 \\
\hline & Rio de Janeiro & 01 & 2,05 \\
\hline & Santa Catarina & 01 & 2,05 \\
\hline & Tocantins & 01 & 2,05 \\
\hline & Não informado ou outro & 02 & 4,10 \\
\hline \multicolumn{4}{|c|}{ Tipos de exercícios físicos } \\
\hline & Caminhada & 14 & 28,55 \\
\hline & Alongamento e hidroginástica & 03 & 6,10 \\
\hline & Musculação & 03 & 6,10 \\
\hline & Bicicleta & 02 & 4,10 \\
\hline & Caminhada e bicicleta & 01 & 2,05 \\
\hline
\end{tabular}

\begin{tabular}{|c|c|c|c|}
\hline & Caminhada e academia de rua & 01 & 2,05 \\
\hline & Não informado & 25 & 51,05 \\
\hline \multicolumn{4}{|c|}{ Região Brasileira } \\
\hline & Nordeste & 25 & 51,00 \\
\hline & Sudeste & 12 & 24,50 \\
\hline & Centro-oeste & 7 & 14,30 \\
\hline & Norte & 2 & 4,10 \\
\hline & Sul & 1 & 2,05 \\
\hline & Não informado & 2 & 4,05 \\
\hline Total & & 49 & 100,00 \\
\hline
\end{tabular}

Na categoria analítica renda financeira, Veras et al. (2001) sustentam que os idosos das regiões Sul, Sudeste e Centro-Oeste apresentam uma distribuição de renda mais elevada e bastante desigual, em comparação as outras regiões.

Nesse sentido, além do fator renda financeira, outros fatores como a melhoria da escolaridade, também, explicam as mudanças, inclusive, no que se refere aos arranjos habitacionais (CAMARGOS; MACHADO; RODRIGUES, 2007).

Já na categoria que analisou a questão gênero e, conforme os estudos desenvolvidos por Pinheiro et al (2002), as taxas de procura por serviços de saúde entre os sexos são altas entre os idosos. Nesse sentido e segundo o autor em questão, as mulheres buscam mais serviços para realização de exames de rotina e prevenção, enquanto os homens procuram serviços de saúde predominantemente por motivo de doença.

Já segundo outros pesquisadores, um dos fatores da maior procura da mulher aos serviços de saúde é a questão cultural ou social, em que a mulher, normalmente, é responsável por acompanhar os filhos e os idosos a consultas e frequentar o pré-natal, o que a torna mais suscetível a utilização dos serviços de saúde (VENTURI et al., 2008).

$\mathrm{Na}$ categoria que analisou a questão do tabagismo juntamente a pessoas idosas, Paskulini e Vianna (2007) apontam que a redução do tabagismo entre idosos significa redução do número total de idosos fumantes, fortalecendo a importância dos programas de cessamento do tabagismo para qualquer grupo etário.

A combinação entre o grau de dependência à nicotina e a motivação para o cessamento do tabagismo apresentaram relevância estatística, pois tem sido ressaltado que a motivação individual é um dos fatores mais relevantes para a cessação definitiva do tabagismo, relacionando com uma gama de variáveis hereditárias, psicológicas, fisiológicas e ambientais (MIRAGAYA et al., 2013). 
Na categoria etilismo, a literatura científica informa que o número de idosos que não fazem o uso de bebida alcoólica aumentou, podendo ter relação com as campanhas que buscam influenciar as pessoas por meio da informação, difundindo na mídia impressa, televisiva e até eletrônica, os problemas de saúde advindos do consumo de bebida alcoólica e a direção de um automóvel podem causar (CAMARGO et al., 2011)

Já na categoria que analisou o nível educacional dos sujeitos da pesquisa, Mastroeni et al. (2007) defendem que, atualmente, os idosos economicamente ativos acabam sendo pressionados pelo sistema competitivo do mercado de trabalho, principalmente no que diz respeito ao baixo grau de escolaridade que a maioria apresenta o que dificulta a busca por melhores salários.

Já em outros estudos, o significativo número de pessoas idosas com pouco ou nenhum grau de escolaridade, é considerado com um na realidade dos países em desenvolvimento como o Brasil, principalmente quando se trata de idosos que viveram sua infância em época em que o ensino não era prioridade (HOTT; PIRES, 2011).

Já na categoria que analisou a questão da prática de exercícios físicos, a literatura científica aponta que, os indivíduos sedentários apresentam nível inferior de autonomia para o desempenho nas AVDs e AIVDs quando comparados aos indivíduos ativos fisicamente, tendo maior incidência entre os idosos avançados. Já para outros pesquisadores, o sedentarismo pode ser, isoladamente, responsável por grave estado de limitação da saúde do idoso, mormente os mais longevos (BORGES; MOREIRA, 2009).

Apesar das evidências acumuladas sobre os benefícios da atividade física, seus níveis tendem a declinar com o avanço da idade, pois, mesmo sabendo a importância da atividade física, raramente é suficiente para motivar um indivíduo sedentário a iniciar e realizá-la de forma adequada e regularmente. Há fatores como desculpas que dificultam a tomada de decisão do indivíduo no sentido de iniciar alguma atividade física e problemas de saúde como impedimento para o exercício (VALERIO; RAMOS, 2013).

$\mathrm{Na}$ categoria analítica tipos de exercícios físicos, esta se encontra de comum acordo com a literatura científica que defende que as atividades mais realizadas e as que causam maior satisfação aos idosos são ginásticas e exercícios físicos diversos, seguida de atividades na academia como musculação (FREIRE et al., 2015).
Nesse sentido, e com o passar dos anos, os espaços e equipamentos públicos de lazer das cidades vêm se caracterizando, gradativamente, como locais constantes de escolha dos idosos para a realização das mais diversas atividades tais como caminhadas, ginástica ao ar livre, corridas, entre outros. Entretanto, a existência desses espaços vem sendo comprometida devido ao processo de urbanização crescente, dificultando, assim, a qualidade de vida (QV) das pessoas que utilizam tais locais (FONSECA; NÓBREGA, 2012).

$\mathrm{Na}$ categoria que analisou a existência de processos alérgicos junto às pessoas idosas, segundo alguns autores, a maior parte dos idosos apresenta um conhecimento inadequado sobre a influenza, pois relatam ter sintomatologia de alergias e mesmo de resfriado comum quando manifestações clínicas relatadas são de influenza. Dessa forma, alguns desses idosos comparam os episódios de resfriado com alergias, devido à mudança repentina de tempo (SANTOS; OLIVEIRA, 2010).

Nesse sentido e, nos últimos anos, ocorreu um aumento progressivo de doenças alérgicas na população idosa em todo o mundo. Além disto, vários fatores em idosos contribuem para o risco de desenvolvimento de alergias, e que estão relacionados às suas condições, incluindo fragilidade, coexistência de outras patologias e a polifarmácia. Os principais tipos de alergias encontradas em idosos são rinite, asma, dermatite de contato, urticária e hipersensibilidade medicamentosa (ALVES; CALAMITA; CALAMITA, 2014).

Já na categoria que analisou o consumo de adoçantes por idosos, é percebida uma tendência de crescimento da prevalência de uso de adoçante dietético conforme o aumento da idade (ZANINI, 2010; ARAÚJO et al., 2010).

Assim, a dieta para o idoso deveria apresentar características como fácil preparação, estimulante do apetite para que ele tenha vontade de comer sozinho, variada e bem apresentada, de fácil mastigação e digestão equilibrada para melhorar a qualidade de vida (QV) além de, abundância e frequência (PLASTER, 2006).

$\mathrm{Na}$ categoria que analisou a questão da restrição alimentar em pessoas idosas, estudos apontam que pacientes idosos podem apresentar lesões decorrentes da dermatite atópica. Os testes cutâneos de leitura imediata para aeroalérgenos e alimentos são mais sensíveis quando comparados à dosagem sérica de IgE específica. A sensibilização a alérgenos alimentares foi mais precoce em relação aos dados da literatura, destacando-se o leite, ovo,

1 
camarão, banana, milho e amendoim (TASSI et al., 2007).

$\mathrm{Na}$ categoria que se debruçou em analisar o sono do idoso, estudos apontam que esse quantitativo se dá em virtude da grande quantidade desses idosos usarem medicamentos, serem ansiosos, possuírem doenças e das mudanças decorrentes ao processo fisiológico do envelhecimento (PLASTER, 2006).

Já para outros pesquisadores, estudos epidemiológicos apontam que, no Brasil, a prevalência é de $50 \%$ de transtornos do sono em pessoas idosas. Esses transtornos do sono estão associados à diminuição da saúde física, cognitiva e emocional, podendo implicar a diminuição do senso de bem-estar, dificuldade no exercício de funções sociais, depressão e diminuição da qualidade de vida e, ainda, um aumento do potencial para morbidades e mortalidade (OLIVEIRA et al., 2010).

Assim, pessoas idosas relatam apresentar mais despertares durante a noite e há um aumento significativo da queixa de insônia. Os idosos queixam-se, principalmente, sobre hipersonia ou insônia, as quais frequentemente são secundárias a outras doenças. Entretanto, a maioria dos idosos tem queixas relacionadas ao sono decorrentes de mudanças fisiológicas específicas do processo de envelhecimento ou de doenças que podem causar distúrbios secundários de sono (CORREA; CEOLIM, 2008).

Já na categoria região administrativa em que o idoso é proveniente, é identificado segundo a literatura que as participações de idosos na população total do Distrito Federal (DF) são verificadas nas regiões administrativas (RAs) mais estáveis, com renda mais elevada como o Lago Sul $(30,1 \%)$, o Plano Piloto $(21,9 \%)$ e o Lago Norte $(19,8 \%)$, seguidas de Águas Claras (10,5\%) e Sudoeste $(11,9 \%)$, muito embora sejam regiões de alta renda, apresentavam baixos percentuais de população idosa, foram destacados percentuais elevados de idosos observados no Gama (18,5\%) e em Taguatinga (18,3\%), que são RAs consideradas de renda média e mais antigas (DISTRITO FEDERAL, 2013).

Dessa forma, o número de idosos é maior na população do Lago Sul, onde há, aproximadamente, 20\% da população total. As menores porcentagens aparecem no SIA, Estrutural, Itapoã, Varjão e São Sebastião, onde a porcentagem de idosos é menor que 3\% (OLIVEIRA, 2014).

$\mathrm{Na}$ categoria que analisou a questão do quantitativo de filhos, segundo alguns estudos, entre 1990 e 2030, a população idosa brasileira será composta, progressiva- mente, por gerações de pais idosos que geraram o declínio da fecundidade, ou seja, será formada por pessoas idosas com um número cada vez menor de filhos (CAMARGOS; MACHADO; RODRIGUES, 2007).

Já na tabela de número 02, é possível verificar que a maior frequência das pessoas idosas analisadas, ou seja, 95,90\% ( $n=47$ ) obtiveram pontuação 06, se apresentando independência em sua funcionalidade e realização de atividades de vida diária (AVD) e atividades instrumentais de vida diária (AIVD).

Tabela 3 - Pontuação adquirida pelos idosos atendidos pela CACUniCEUB por meio da utilização do Índice de Katz no ano de 2015, Brasília, Distrito Federal (D.F.), Brasil ( $\mathrm{n}=49)$ :

\begin{tabular}{lccc}
\hline Pontuação adquirida & Frequência & $\%$ & Avaliação \\
\hline 06 & 47 & 95,90 & Independência \\
05 & 02 & 4,10 & Independência \\
\hline Total & $\mathbf{4 9}$ & $\mathbf{1 0 0 , 0 0}$ & \\
\hline Fonte: Produção dos autores, 2016. & &
\end{tabular}

Nesse sentido, manter os idosos independentes funcionalmente é o primeiro passo para se atingir uma melhor qualidade de vida. Para tanto, é necessário o planejamento de programas específicos de intervenção, visando à eliminação de fatores de riscos relacionados com a incapacidade funcional. Ao lado disso, devem ser elaboradas ações de promoção da saúde, prevenção de doenças, recuperação e reabilitação, que interfiram, diretamente, na manutenção da capacidade funcional desses idosos. Deve-se levar em conta que essa capacidade funcional depende, também, de fatores demográficos, socioeconômicos, culturais e psicossociais, além do estilo de vida (FERREIRA et al., 2012).

Dessa forma, em relação à funcionalidade para as atividades de vida diária, mais de $60 \%$ dos idosos do estudo foram classificados como independentes, no entanto, outra parte relatou precisar de ajuda parcial para realizar as AVD. O resultado tem como importância o declínio hierárquico da funcionalidade dos idosos, que se demonstra, inicialmente, pelo compromisso de exercícios físicos, intelectuais e sociais mais complexas e indicativas de autonomia, independência e uma vida saudável, prosseguindo, sucessivamente, para as tarefas voltadas ao autocuidado e à sobrevivência, mais rotineiras e executadas em ambiente mais restrito (PAULA et al., 2005).

É de extrema importância a promoção da saúde no ensinamento da realização de atividade física durante o processo de envelhecimento pelos Enfermeiros de Reabilitação, pois é um aspeto primordial para um envelheci- 
mento saudável no cuidado da saúde e da funcionalidade desses idosos. Assim, se tornando indispensável à realização das AVD e AIVD de forma independente e na prevenção de doença e incapacidades (FERNADES, 2015).

\section{Conclusão}

O presente estudo demonstrou que as pessoas idosas atendidas no CAC-UniCEUB são independentes no que se refere a sua funcionalidade. Apesar de a maioria das pessoas idosas analisadas no presente estudo residirem em cidades satélites ou regiões administrativas diferentes daquela onde se encontra instalado o CAC-UniCEUB, estas são favorecidas, economicamente, por residirem próximo a capital federal do Brasil.

Essa constatação aponta para o favorecimento destes economicamente, principalmente conforme evidenciada na categoria analítica renda financeira. Outro fator que contribui para a independência da funcionalidade dos idosos analisados é a escolaridade de estes ser, em sua maioria, constituída por pessoas que declararam possuir ensino fundamental ou médio, facilitando uma maior autonomia da pessoa idosa.

A qualidade de vida verificada pela alimentação saudável, não consumo de bebida alcoólica, tabaco, ausência de alergias e realização de atividades de vida diária e atividades instrumentais de vida diária, também, contribuem para que os idosos avaliados apresentem independência em sua funcionalidade.

Foi observado que nenhum idoso é natural de Brasília e que a região predominante dessa população é a região nordeste, devido à historicidade e à busca por melhores condições de vida na capital federal. Com isso podemos concluir que $100 \%$ dessa população é independente, atribuindo a melhores políticas públicas de saúde.

É de extrema importância o papel do enfermeiro e dos profissionais da saúde no CAC-UniCEUB, pois, mesmo que tenhamos percebido neste estudo que $100 \%$ desses idosos são independentes, é importante orientarmo-nos quanto às limitações relacionado às patologias, os agravos, restrições e limitações com o passar do tempo, orientando e tirando dúvidas, assim, proporcionando uma melhor qualidade de vida melhor para esses idosos e sua família.

\section{Referências}

ALVES, L. C. et al. A influência das doenças crônicas na capacidade funcional dos idosos do Município de São Paulo, Brasil. Cadernos de Saúde Pública, Rio de Janeiro, v. 23, n. 8, p. 1924-1930, ago. 2007.
ALVES, L. D. S.; CALAMITA, A. B. P.; CALAMITA, Z. Estudo comparativo sobre a prevalência de alergias entre idosos e não idosos. Brazilian Journal Allergy and Immunology, São Paulo, v. 2, n. 2, p. 75-80, abr. 2014.

ARAÚJO, I. F. L. et al. Análise comparativa da capacidade funcional entre idosos institucionalizados e não institucionalizados. Estudos, Goiânia, v. 37, n. 1/2, p. 101-111, jan./fev. 2010.

BORGES, M. R.; MOREIRA, A. K. Influências da prática de atividades físicas na terceira idade: estudo comparativo dos níveis de autonomia para o desempenho nas AVDs e AIVDs entre idosos ativos fisicamente e idosos sedentários. Motriz, Rio Claro, v. 15, n. 3, p. 562-573, jul./ set. 2009.

BRASIL. Lei $\mathbf{n}^{\circ} \mathbf{1 0 . 7 4 1}$, de $1^{\circ}$ de outubro de 2003. Estatuto do Idoso, Brasília, 2003. Disponível em: <http:// www.planalto.gov.br/ccivil_03/leis/2003/L10.741.htm>. Acesso em: 14 ago. 2015.

BRASIL. Lei no $\mathbf{n}^{\mathbf{8}} \mathbf{8 4 2}$, de 4 de janeiro de 1994. Política Nacional de Saúde da Pessoa Idosa, Brasília, 1994. Disponível em: <http://www.planalto.gov.br/CCivil_03/leis/ L8842.htm>. Acesso em: 13 ago. 2015.

BRASIL. Ministério da Saúde. Atenção à saúde da pessoa idosa e envelhecimento. Brasília: Ministério da Saúde, 2010 .

BRASIL. Presidência da República. Casa Civil. Sub-Secretaria para Assuntos Jurídicos. Lei 10.741 de $1^{\circ}$ de Outubro de 2003. Dispõe sobre o Estatuto do Idoso e dá outras providências. Disponível em: <http://www.planalto. gov.br/ccivil_03/leis/2003/110.741.htm>. Acesso em: 13 ago. 2015 .

CAMARA, F. M. et al . Capacidade funcional do idoso: formas de avaliação e tendências. Acta Fisiátrica, São Paulo, v. 15, n. 4, p. 249-256, mar./nov. 2008.

CAMARGO, B. V. et al. Representações sociais de saúde e cuidado: um estudo multicêntrico sobre vulnerabilidade masculina. Temas em Psicologia, Ribeirão Preto, v. 19, n. 1, p. 179-192, jun. 2011.

CAMARGOS, M. C. S.; MACHADO, C. J.; RODRIGUES, R. N. A relação entre renda e morar sozinho para idosos paulistanos - 2000. Revista Brasileira de Estudos de População, Rio de Janeiro, v. 24, n. 1, p. 37-51, jan./jun. 2007.

CORRÊA, K; CEOLIM, M. F. Qualidade do sono em pacientes idosos com patologias vasculares periféricas. Revista da Escola de Enfermagem da USP, São Paulo, v. 42, n. 1, p. 12-18, mar. 2008.

COSTA, E. C.; NAKATANI, A. Y. K.; BACHION, M. M. 
Capacidade de idosos da comunidade para desenvolver atividades de vida diária e atividades instrumentais de vida diária. Acta Paulista de Enfermagem, São Paulo, v. 19, n. 1, p. 43-48, mar. 2006.

DISTRITO FEDERAL. Companhia de Planejamento do Distrito Federal. Perfil dos idosos no Distrito Federal, segundo as regiões administrativas. Brasília: CODEPLAN, 2013. Disponível em: <http://www.codeplan.df.gov.br/images/CODEPLAN/PDF/Pesquisas $\% 20$ Socioecon\%C3\%B4micas/2013/PERFIL\%20DO\%20 IDOSO\%20NO\%20DF.pdf>. Acesso em: 22 abr. 2016.

DUARTE, Y. A. O.; ANDRADE, C. L.; LEBRÃO, M. L. O Índex de Katz na avaliação da funcionalidade dos idosos. Revista da Escola de Enfermagem USP, São Paulo, v. 41, n. 2, p. 317-325, jun. 2007.

FERNANDES, C. N. Funcionalidade, aptidão física e qualidade de vida do idoso da região Minho-Lima: contributos para a enfermagem de reabilitação. 149 f. Dissertação (Mestrado) - Instituto Politécnico de Viana do Castelo, Alto Minho, 2015.

FERREIRA, O. G. L. et al . Envelhecimento ativo e sua relação com a independência funcional. Texto e Contexto Enfermagem, Santa Catarina, v. 21, n. 3, p. 513-518, jul./ set. 2012.

FERREIRA, O. G. L. et al. O envelhecimento ativo sob o olhar de idosos funcionalmente independentes. Revista da Escola de Enfermagem USP, São Paulo, v. 44, n. 4, p. 1065-1069, dez. 2010.

FONSECA, I. L.; NÓBREGA, W. R. M. Lazer e terceira idade: um estudo acerca dos espaços e equipamentos públicos no município de Parelhas/RN. Revista Turismo Estudos e Práticas, Rio Grande do Norte, v. 1, n. 2, p. 61-79, jul./dez. 2012.

FREIRE, G. V. et al. Perfil de idosos que frequentam um centro de convivência da terceira idade. Revista Interdisciplinar, Piauí, v. 8, n. 2, p. 11-19, abr./jun. 2015.

GUERRA, A. C. L. C.; CALDAS, C. P. Dificuldades e recompensas no processo de envelhecimento: a percepção do sujeito idoso. Ciência \& Saúde Coletiva, Rio de Janeiro, v. 15, n. 6, p. 2931-2940, set. 2010.

HOTT, A. M.; PIRES, V. A. T. N. Perfil dos idosos inseridos em um centro de convivência. Revista Enfermagem Integrada, Minas Gerais, v. 4, n. 1, p. 765- 778, jul./ago. 2011.

KARSCH, U. M. Idosos dependentes: famílias e cuidadores. Caderno de Saúde Pública, Rio de Janeiro, v. 19, n. 3, p. 861-866, jun. 2003.

KATZ, S. et al. Studies of illness in the aged. The index of
ADL: a standardized measure of biological ans psychosocial function. JAMA, New York, v. 185, n. 12, p. 914-919, set. 1963.

KATZ, S.; STROUD III, M. W. Functional assessment in geriatrics: a review of progress and directions. Journal of the American Geriatrics Society, Malden, v. 37, n. 3, p. 267-71, ago. 1989.

LOURENÇO, R. A.; VERAS, R. P. Mini-exame do estado mental: características psicométricas em idosos ambulatoriais. Revista de Saúde Pública, São Paulo, v. 40, n. 4, p. 712-719, ago. 2006.

MASTROENI, M. F. et al. Perfil demográfico de idosos da cidade de Joinville, Santa Catarina: estudo de base domiciliar. Revista Brasileira de Epidemiologia, São Paulo, v. 10, n. 2, p. 190-201, jun. 2007.

MIRAgAYA, J. et al. Perfil dos idosos no Distrito Federal, segundo as regiões administrativas. Brasília: CODEPLAN, 2013.

MOREIRA, R. S. et al. A saúde bucal do idoso brasileiro: revisão sistemática sobre o quadro epidemiológico e acesso aos serviços de saúde bucal. Cadernos de Saúde Pública, Rio de Janeiro, v. 21, n. 6, p. 1665-1675, dez. 2005.

NAKATANI, A. Y. K. et al. Capacidade funcional em idosos na comunidade e propostas de intervenções pela equipe de saúde. Revista Eletrônica de Enfermagem, Goiânia, v. 11, n. 1, p. 144-150, jan./mar. 2009.

OLIVEIRA, A. B. Avaliação da pele em idosos de um hospital universitário do Distrito Federal: ocorrência de alterações. 28 f. Monografia (Graduação) - Faculdade de Enfermagem, Universidade de Brasília, Brasília, 2014.

OLIVEIRA, B. H. D. et al. Relações entre padrão do sono, saúde percebida e variáveis socioeconômicas em uma amostra de idosos residentes na comunidade - Estudo PENSA. Ciência e Saúde Coletiva, Rio de Janeiro, v. 15, n. 3, p. 851-860, maio 2010.

ORGANIZAÇÃO MUNDIAL DE SAÚDE. Envelhecimento ativo: uma política de saúde. Brasília: OPAS, 2005.

PARAHYBA, M. I.; SIMÕES, C. C. S. A prevalência de incapacidade funcional em idosos no Brasil. Ciência \& Saúde Coletiva, Rio de Janeiro, v. 11, n. 4, p. 967-974, dez. 2006.

PASKULIN, L. M. G.; VIANNA, L. A. C. Perfil sociodemográfico e condições de saúde auto-referidas de idosos de Porto Alegre. Revista de Saúde Pública, São Paulo, v. 41, n. 5, p. 757-768, out. 2007.

PAULA, A. F. M. et al. Perfis de funcionalidade e relação com óbito em idosos assistidos em serviço ambulatorial 
de geriatria. Caderno de Terapia Ocupacional UFSCar, São Carlos, v. 23, n. 1, p. 153-162, jan./mar. 2015.

PEDRAZZI, E. C.; RODRIGUES, R. A. P.; SCHIAVETO, F. V. Morbidade referida e capacidade funcional de idosos. Ciência, Cuidado Saúde, Maringá, v. 6, n. 4, p. $407-$ 413, out./dez. 2007.

PELEGRIN, A. K. A. P. et al. Idosos de uma instituição de longa permanência de Ribeirão Preto: níveis de capacidade funcional. Arquivo em Ciências da Saúde, São José do Rio Preto, v. 15, n. 4, p. 182-188, out./dez. 2008.

PEREIRA, G. N. et al. Indicadores demográficos e socioeconômicos associados à incapacidade funcional em idosos. Cadernos de Saúde Pública, Rio de Janeiro, v. 28, n. 11, p. 2035-2042, nov. 2012.

PINHEIRO, R. S. et al. Gênero, morbidade, acesso e utilização de serviços de saúde no Brasil. Ciência \& Saúde Coletiva, Rio de Janeiro, v. 7, n. 4, p. 687-707, nov. 2002.

PINHO, T. A. M. et al. Avaliação do risco de quedas em idosos atendidos em Unidade Básica de Saúde. Revista da Escola de Enfermagem da USP, São Paulo, v. 46, n. 2, p. 320-327, abr. 2012.

PLASTER, W. Adesão ao tratamento da hipertensão arterial por idosos usuários da Unidade Básica de Saúde Princesa Isabel em Cacoal - RO. 89 f. Dissertação (Mestrado) - Programa Multiinstitucional de Pós- Graduação em Ciências da Saúde, Convênio Rede Centro Oeste (UnB/ UFG/ UFMS), Goiânia, 2006.

RODRIGUES, K. J. et al. Avaliação da capacidade funcional de idosos pertencentes à área de abrangência de uma ESF-Cáceres - MT. Revista Eletrônica Gestão \& Saúde, Brasília, v. 4, n. 3, p. 747-761, set. 2013.
SANTOS, M. I. P. O.; GRIEP, R. H. Capacidade funcional de idosos atendidos em um programa do SUS em Belém (PA). Ciência \& Saúde Coletiva, Rio de Janeiro, v. 18, n. 3, p. 753-761, mar. 2013.

SANTOS, Z. M. G.; OLIVEIRA, M. L. C. Avaliação dos conhecimentos, atitudes e práticas dos idosos sobre a vacina contra a Influenza, na UBS, Taguatinga, DF, 2009*. Epidemiologia e Serviços de Saúde, Brasília, v. 19, n. 3, p. 205-216, set. 2010.

TASSI, R. O.S. et al. Perfil de sensibilização a aeroalérgenos e alimentos em pacientes com dermatite atópica do HSPE-SP. Revista Brasileira de Alergia e Imunopatologia, São Paulo, v. 30, n. 3, p. 101-106, maio 2007.

VALERIO, M. P.; RAMOS, L. R. Promoção de atividade física à população idosa: revisando possibilidades. Revista Didática Sistêmica, Rio Grande, v. 15, n. 2, p. 155 173, jul./dez. 2013.

VENTURI, I. et al. Identificação da área de influência do serviço de atenção básica do sistema público de saúde à população idosa, município de Viçosa - MG. Ciência e Saúde Coletiva, Rio de Janeiro, v. 13, n. 4, p. 1293-1304, ago. 2008.

VERAS, R. et al. Velhice numa perspectiva de futuro saudável. Rio de Janeiro: UERJ, UnATI, 2001.

ZANINI, R. V. Prevalência de utilização de adoçantes dietéticos: um estudo de base populacional. $123 \mathrm{f}$. Dissertação (Mestrado) - Programa de Pós-Graduação em Epidemiologia, Universidade Federal de Pelotas, Pelotas, 2010. 